Pacific Journal of Mathematics

THE BOUNDARY MODULUS OF CONTINUITY OF HARMONIC 


\section{THE BOUNDARY MODULUS OF CONTINUITY OF HARMONIC FUNCTIONS}

\section{Elgin H. Johnston}

Let $G$ be a bounded domain in the complex plane and let $u(z)$ be continuous on $\bar{G}$. In this paper we study the boundary modules of continuity, $\tilde{\omega}(\delta)$, of $u$ on $\partial G$ and the modulus of continuity, $\omega(\delta)$, of $u$ on $\bar{G}$. We investigate the extent to which the inequality " $\omega(\delta) \leq \tilde{\omega}(\delta)$ " holds when $u$ is harmonic on $G$ and show that the precise formulation of such inequalities depends on the smoothness of $\partial G$.

1. Introduction. Let $G$ be a bounded domain in the complex plane and let $u(z)$ be continuous on $\bar{G}$. The modulus of continuity (MOC) of $u(z)$ on $\bar{G}$ is the function $\omega_{u}(\delta, \bar{G})$ defined for $\delta \geqq 0$ by

$$
\omega_{u}(\delta, \bar{G})=\sup \left\{\left|u(z)-u\left(z^{\prime}\right)\right|: z, z^{\prime} \in \bar{G},\left|z-z^{\prime}\right| \leqq \delta\right\} .
$$

Thus $\omega_{u}(\delta, \bar{G})$ is nondecreasing and $\lim _{\bar{\delta} \rightarrow 0^{+}} \omega(\delta)=\omega(0)=0$. If $\bar{G}$ is, say, convex, then $\omega_{u}(\delta)$ is subadditive and continuous. The boundary modulus of continuity (BMOC) is denoted $\tilde{\omega}_{u}(\delta, \partial G)$ and defined by

$$
\tilde{\omega}_{u}(\delta, \partial G)=\sup \left\{\left|u(\zeta)-u\left(\zeta^{\prime}\right)\right|: \zeta, \zeta^{\prime} \in \partial G,\left|\zeta-\zeta^{\prime}\right| \leqq \delta\right\} .
$$

When no confusion should arise, we will simply write $\omega(\delta)$ and $\tilde{\omega}(\delta)$.

It is clear that $\tilde{\omega}_{u}(\delta, \partial G) \leqq \omega_{u}(\delta, \bar{G})(\delta \geqq 0)$, and that if $u(z)$ is simply continuous on $\bar{G}$, little more can be said. In this paper we investigate the extent to which the reverse inequality holds for $u(z)$ harmonic (or analytic) on $G$.

Rubel, Taylor and Shields [6, p. 31] have proved the following result for $u$ analytic.

THEOREM. Let $G$ be simply connected and let $\phi(\delta)(\delta \geqq 0)$ be a continuous increasing, nonnegative subadditive function. Then for $u(z)$ analytic on $G$, continuous on $\bar{G}$,

$$
\tilde{\omega}(\delta) \leqq \phi(\delta) \Longrightarrow \omega(\delta) \leqq C \dot{\phi}(\delta),
$$

where $C$ is an absolute constant, independent of $G$.

It can be shown that $C>1$ is necessary.

For $u(z)$ harmonic, it is known that if $G=D=\Delta(0,1)$ is the unit disk and $u(z)$ is harmonic on $D$, continuous on $\bar{D}$, then

$$
\omega(\delta) \leqq C\left(\log \frac{1}{\delta}\right) \tilde{\omega}(\delta) \quad\left(0<\delta \leqq \frac{1}{2}\right)
$$


where $C$ is an absolute constant. This result is best possible on $D$ in the sense that the $\log (1 / \delta)$ factor cannot be improved [6, p. 34]. We add, however, that (1) can be sharpened for some $\tilde{\omega}_{u}$ since standard techniques for estimating Poisson integrals give

$$
\omega(\delta) \leqq 3 \tilde{\omega}(\delta)+\frac{\pi \delta}{4} \int_{\delta}^{\pi} \frac{\tilde{\omega}(s)}{s^{2}} d s .
$$

It can be shown that (1) follows from (2). We note that (2) also gives a result of Hardy and Littlewood [3]: if $\tilde{\omega}(\delta) \leqq \delta^{\alpha}(0<\alpha<1)$, then $\omega(\delta) \leqq C \delta^{\alpha}$. More recently Dankel [2] has shown that (1) holds for a wider class of bounded simply connected domains $G$. In particular, (1) holds if $\partial G$ is an analytic curve or if $\partial G$ is Dinismooth and has bounded arc chord ratio.

In this paper we answer some of the remaining open questions concerning the relation between $\omega_{u}(\delta)$ and $\tilde{\omega}_{u}(\delta)$ for harmonic $u$. In $\S 2$ we show that the relation between the MOC and BMOC is related to the smoothness of $\partial G$, and describe a wider class of domains $G$ for which (1) and (2) hold. In $\S 3$ we consider a function $f=$ $u+i v$ analytic on $G$ and briefly discuss the relationship between $\tilde{\omega}_{u}(\delta)$ and $\omega_{f}(\delta)$. In $\S 4$ we give a class of examples showing the results of $\S 2$ are best possible and at the same time answer a question of Dankel [2] by showing (1) is not valid on arbitrary bounded, simply connected domains.

2. The MOC of harmonic functions. The proofs of the main theorems in this section use the following result of A. Beurling [1, p. 55].

THEOREM. Let $G$ be a simply connected domain in the complex plane, let $\gamma \subseteq \partial G$ and let $z \in G$. Let $d(z, \partial G)$ and $d(z, \gamma)$ denote the distance from $z$ to $\partial G$ and $\gamma$ respectively, and $\mu(z, \gamma, G)$ denote the harmonic measure of $\gamma$ with respect to $z$ and $G$. Then

$$
\mu(z, \gamma, G) \leqq \frac{4}{\pi} \operatorname{Arctan}\left(\frac{d(z, \partial G)}{d(z, \gamma)}\right)^{1 / 2} \leqq \frac{4}{\pi}\left(\frac{d(z, \partial G)}{d(z, \gamma)}\right)^{1 / 2},
$$

where the last inequality follows since Arc $\tan x \leqq x$ for $x \geqq 0$. We can now prove the following theorem.

THEOREM 1. Let $G$ be a bounded simply connected domain and suppose $u(z)$ is harmonic on $G$ and continuous on $\bar{G}$. Then

$$
\omega(\delta) \leqq \tilde{\omega}(2 \delta)+\frac{8 \sqrt{2}}{\pi \log 2} \delta^{1 / 2} \int_{\delta}^{|G|} \frac{\tilde{\omega}(s)}{s^{3 / 2}} d s,
$$


where $|G|$ denotes the diameter of $G$.

Proof. We first observe that

$$
\begin{aligned}
\omega(\delta) & =\sup \left\{\left|u(z)-u\left(z^{\prime}\right)\right|: z, z^{\prime} \in \bar{G},\left|z-z^{\prime}\right| \leqq \delta\right\} \\
& =\sup \{|u(\zeta)-u(z)|: \zeta \in \partial G, z \in \bar{G},|z-\zeta| \leqq \delta\}
\end{aligned}
$$

This equality is proved in $[6$, p. 26] for analytic $u$ and the same proof is valid for harmonic $u$. We assume $z \in \bar{G}, \zeta \in \partial G$ have been chosen with $|z-\zeta| \leqq \delta$ and $|u(z)-u(\zeta)|=\omega(\delta)$. Without loss of generality, we assume $\zeta=0$. If $z \in \partial G$, then $\omega(\delta)=\tilde{\omega}(\delta)$ gives the desired inequality. We assume $z \in G$. Then

$$
\begin{aligned}
\omega(\delta)=|u(z)-u(0)| & =\left|\int_{\partial G}\{u(\zeta)-u(0)\} \mu(z, d \zeta, G)\right| \\
& \leqq \int_{\partial G} \tilde{\omega}(|\zeta|) \mu(z, d \zeta, G) .
\end{aligned}
$$

Let $A_{1}=\{\zeta \in \partial G:|\zeta| \leqq 2 \delta\}$ and $A_{n}=\left\{\zeta \in \partial G: 2^{n-1} \delta<|\zeta| \leqq 2^{n} \delta\right\} \quad(2 \leqq$ $n \leqq N=1+\left[\log _{2}|G| / \delta\right] \cdots "[]$ " denotes the greatest integer function). Then

$$
\begin{aligned}
\omega(\delta) & \leqq \tilde{\omega}(2 \delta)+\sum_{n=2}^{N} \int_{A_{n}} \tilde{\omega}(|\zeta|) \mu(z, d \zeta, G) \\
& \leqq \tilde{\omega}(2 \delta)+\sum_{n=2}^{N} \tilde{\omega}\left(2^{n} \tilde{\delta}\right) \mu\left(z, A_{n}, G\right) \\
& \leqq \tilde{\omega}(2 \delta)+\frac{4}{\pi} \sum_{n=2}^{N} \tilde{\omega}\left(2^{n} \delta\right)\left(\frac{d(z, \partial G)}{d\left(z, A_{n}\right)}\right)^{1 / 2}
\end{aligned}
$$

by (3). Since $d(z, \partial G) \leqq \delta$ and $d\left(z, A_{n}\right) \geqq 2^{n-2} \delta$, we have

$$
\begin{aligned}
\omega(\delta) & \leqq \tilde{\omega}(2 \delta)+\frac{4}{\pi} \sum_{n=2}^{N} \tilde{\omega}\left(2^{n} \delta\right) 2^{2-n / 2} \\
& \leqq \tilde{\omega}(2 \delta)+\frac{8}{\pi} \int_{1}^{N-1} \frac{\tilde{\omega}\left(2^{t+1} \delta\right)}{2^{t / 2}} d t .
\end{aligned}
$$

The result follows by substituting $s=2^{t+1} \delta$ in the last integral.

Two useful corollaries follow from Theorem 1.

CoROllary 2. If $\tilde{\omega}(\delta)$ is subadditive, then

$$
\omega_{u}(\delta) \leqq C \delta^{-1 / 2} \tilde{\omega}_{u}(\delta),
$$

where $C=C(|G|)$ is a positive constant.

CoROLLARY 3. If $\tilde{\omega}(\delta) \leqq \delta^{\alpha}(0 \leqq \alpha \leqq 1)$, then 


$$
\omega(\delta) \leqq C \begin{cases}\delta^{\alpha} & \left(0<\alpha<\frac{1}{2}\right) \\ \delta^{1 / 2} \log \frac{1}{\delta} & \left(\alpha=\frac{1}{2}, 0<\delta<\frac{1}{2}\right) \\ \delta^{1 / 2} & \left(\alpha \geqq \frac{1}{2}\right) .\end{cases}
$$

Again, $C=C(|G|)$ is a positive constant depending on $|G|$.

Corollary 3 follows by integration. Corollary 2 is proved as follows.

Proof. Since $\tilde{\omega}(\delta)$ is a subadditive modulus of continuity, we can find a continuous, nondecreasing concave function $\lambda(\delta)$ for which

$$
\tilde{\omega}(\delta) \leqq \lambda(\delta) \leqq 2 \tilde{\omega}(\delta) \quad(\delta \geqq 0),
$$

[5, p. 45]. Then $\lambda(\delta) / \delta$ is nondecreasing for $\delta>0$. Thus,

$$
\begin{aligned}
\omega_{u}(\delta) & \leqq 2 \tilde{\omega}(\delta)+C \delta^{1 / 2} \int_{\delta}^{|G|} \frac{\lambda(s)}{s^{3 / 2}} d s \\
& \leqq 2 \tilde{\omega}(\delta)+C \delta^{-1 / 2} \lambda(\delta) \int_{\delta}^{|G|} s^{-1 / 2} d s \\
& \leqq C^{\prime} \delta^{-1 / 2} \tilde{\omega}(\delta) .
\end{aligned}
$$

In $\S 4$ we give an example showing Corollaries 2 and 3 give the best possible order of magnitude.

Theorem 1 can be improved in some cases. In particular, our next result relates the global MOC to the BMOC and the smoothness of $\partial G$. We give a definition to classify boundary smoothness.

Definition 4. For $0<\alpha<1$ and $\varepsilon>0$, let

$$
S(\alpha, \varepsilon)=\left\{z:|\operatorname{Arg}(z)| \leqq \frac{\pi \alpha}{2} \text { and } 0 \leqq \operatorname{Re} z \leqq \varepsilon\right\} .
$$

For $\zeta \in C$ and $\theta$ real,

$$
S(\alpha, \varepsilon, \zeta, \theta)=\zeta+e^{i \theta} S(\alpha, \varepsilon),
$$

is the "cone" $S(\alpha, \varepsilon)$ rotated through angle $\theta$ and translated so its vertex is at $\zeta$. A bounded, simply connected open domain $G$ satisfies a (exterior) cone condition of order $(\alpha, \varepsilon)$ if for each $\zeta \in \partial G$ there exists a real $\theta=\theta(\zeta)$ such that

$$
S(\alpha, \varepsilon, \zeta, \theta) \cap G=\phi .
$$

THEOREM 5. Suppose $G$ satisfies a cone condition of order $(\alpha, \varepsilon)$ 
$(0<\alpha<1, \varepsilon>0)$. If $u(z)$ is harmonic on $G$, continuous on $\bar{G}$ and has BMOC $\tilde{\omega}(\delta)$, then

$$
\omega(\delta) \leqq \tilde{\omega}(C \delta)+D \delta^{1 / \beta} \int_{\delta}^{E} \frac{\tilde{\omega}(s)}{s^{(1+\beta) / \beta}} d s
$$

where $\beta=2-\alpha$ and $C, D, E$ are positive constants depending on G.

Proof. We may assume $\zeta=0 \in \partial G, z \in G$ with $|z-\zeta| \leqq \delta$ and $|u(z)-u(\zeta)|=\omega(\delta)$. We further assume $\theta(\zeta)=0$, so that $S(\alpha, \varepsilon, \zeta, \theta)=$ $S(\alpha, \varepsilon)$ and $S(\alpha, \varepsilon) \cap G=\phi$. Let $\eta>0$ and $r>0$ denote, respectively, the center and radius of the circle inscribed in $\partial S(\alpha, \varepsilon)$. We have $d(\eta, \partial G) \geqq r=\varepsilon(1+\csc \alpha / 2)^{-1}$ (see Figure I).

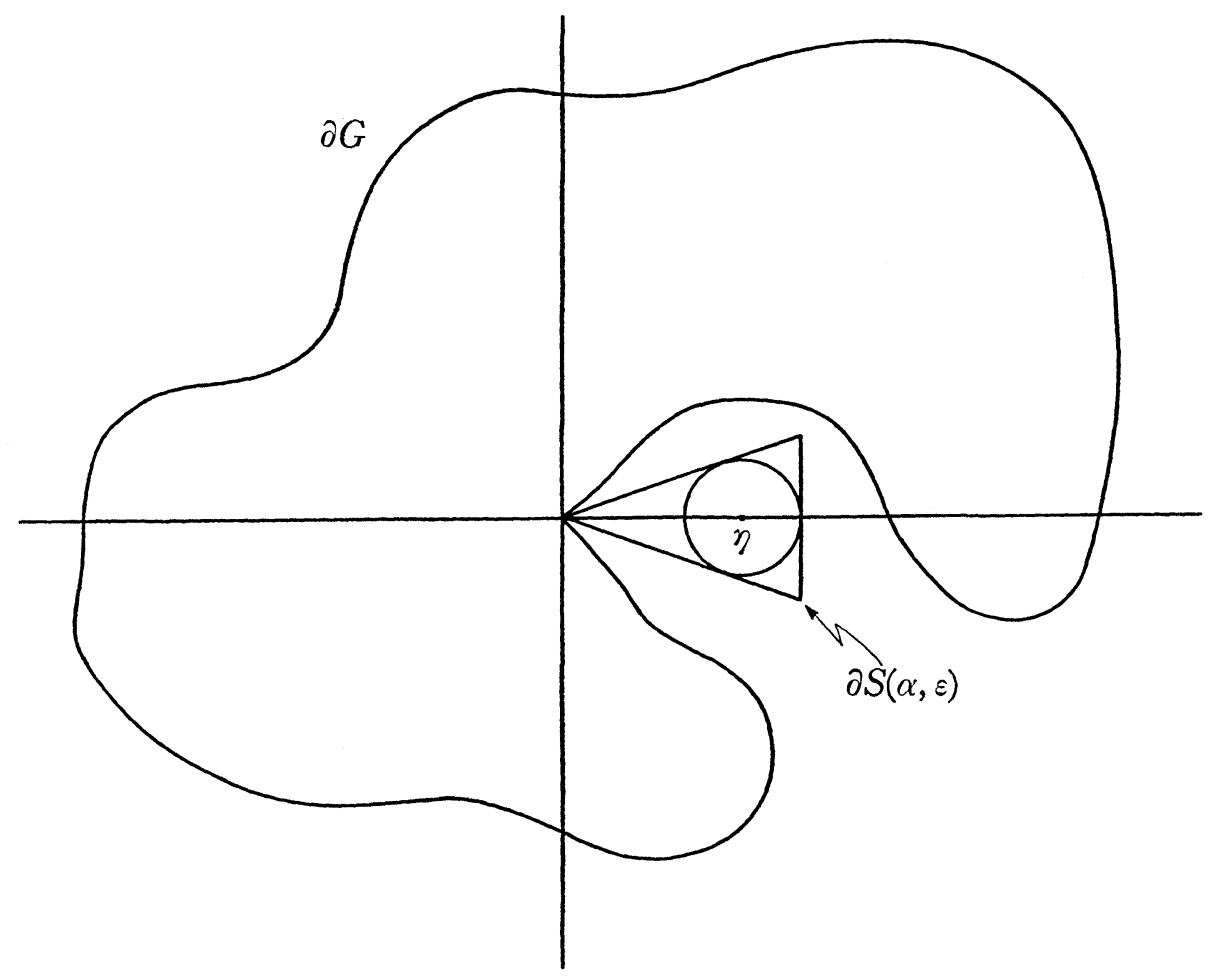

Figure I.

Let

$$
\Phi(z)=\left(\frac{1}{z-\eta}+\frac{1}{\eta}\right)^{2 / \beta} \quad(\beta=2-\alpha),
$$

be a mapping from $G$ in the $z$-plane to a domain $H$ in the $\xi$-plane. We take the branch cut for $\Phi(z)$ along the segment $[0, \eta]$. Then $\Phi$ 
maps $G$ conformally onto $H=\Phi(G)$, with $\Phi(0)=0 \in \partial H$. It is clear, in fact, that $\bar{H} \cap\{\xi<0\}=\phi$, so $\Phi$ is actually one-to-one and continuous on $\bar{G}$. We can now define $v(\xi)=u\left(\Phi^{-1}(\xi)\right)$ for $\xi \in \bar{H}$ by taking the branch cut for

$$
\Phi^{-1}(\xi)=\frac{\eta^{2} \xi^{\beta / 2}}{\eta \xi^{\beta / 2}-1}
$$

along $\{\xi<0\}$. This assures that $\Phi^{-1}$ is analytic on $H$, continuous and one-to-one on $\bar{H}$, with $\bar{G}=\Phi^{-1}(\bar{H})$. Thus $v(\xi)$ is harmonic on $H$ and continuous on $\bar{H}$. We then have

$$
\begin{aligned}
\omega_{u}(\delta, \bar{G}) & =|u(z)-u(0)| \\
& =|v(\Phi(z))-v(0)| \\
& \leqq \int_{\partial H}|v(\xi)-v(0)| \mu(\Phi(z), d \xi, H) \\
& \leqq \int_{\partial H} \tilde{\omega}_{u}\left(\left|\Phi^{-1}(\xi)\right|, \bar{G}\right) \mu(\Phi(z), d \xi, H) .
\end{aligned}
$$

For $\xi \in \bar{H}$ we have $\left|\eta \xi^{\beta / 2}-1\right| \geqq \eta /(\eta+|G|)$. Combining this with (6) and (7) gives

$$
\omega_{u}(\delta, \bar{G}) \leqq \int_{\partial H} \tilde{\omega}_{u}\left(\eta(\eta+|G|)|\xi|^{\beta / 2}\right) \mu(\Phi(z), d \xi, H) .
$$

Let $A_{1}=\left\{\xi \in \partial H:|\xi| \leqq(4 \delta / \eta r)^{2 / \beta}\right\}$ and

$$
A_{n}=\left\{\xi \in \partial H:\left(\frac{2^{n} \delta}{\eta \eta}\right)^{2 / \beta}<|\xi| \leqq\left(\frac{2^{n+1} \delta}{\eta r}\right)^{2 / \beta}\right\} \quad(2 \leqq n \leqq N)
$$

where $N=\left[\log _{2}((\eta+r) / \delta)\right] \leqq \log _{2}(2 \eta / \delta)$. It then follows that

$$
d(\Phi(z), \partial H) \leqq|\Phi(z)| \leqq\left(\frac{\delta}{\eta r}\right)^{2 / \beta},
$$

and

$$
\begin{aligned}
d(\Phi(z), \partial H) & \geqq\left(\frac{2^{n} \delta}{\eta r}\right)^{2 / \beta}-\left(\frac{\delta}{\eta r}\right)^{2 / \beta} \\
& \geqq\left(2^{2 / \beta}-1\right)\left(\frac{2^{n-1} \delta}{\eta r}\right)^{2 / \beta} \quad(2 \leqq n \leqq N) .
\end{aligned}
$$

From (8) and (3) we obtain

$$
\begin{aligned}
\omega(\delta) \leqq \tilde{\omega}\left(\frac{4(\eta+|G|)}{r} \delta\right) & +\frac{4}{\pi} \sum_{n=2}^{N} \tilde{\omega}\left(\frac{2^{n+1}(\eta+|G|)}{r} \delta\right)\left(2^{2 / \beta}-1\right)^{-1 / 2} 2^{(1-n) / \beta} \\
& \leqq \tilde{\omega}(C \delta)+D \int_{1}^{N} \frac{\tilde{\omega}\left(C \delta 2^{t}\right)}{2^{t / \beta}} d t
\end{aligned}
$$


where $C$ and $D$ are positive constants depending on $|G|, \eta$ and $r$. The desired inequality is obtained by letting $s=C \delta 2^{t}$ in the last integral.

We can now write down corollaries similar to those for Theorem 1.

Corollary 6. Assume the hypotheses of Theorem 5. If, in addition, $\tilde{\omega}(\delta)$ is subadditive, then

$$
\omega(\delta) \leqq C \delta^{(1-\beta) / \beta} \tilde{\omega}(\delta),
$$

where $C$ is a positive constant depending on $G$.

CoROLlary 7. Assume the hypotheses of Theorem 5 . If $\tilde{\omega}(\delta) \leqq \delta^{r}(0<\gamma \leqq 1)$, then

$$
\omega(\delta) \leqq C \begin{cases}\delta^{\gamma} & \left(0<\gamma<\frac{1}{\beta}\right) \\ \delta^{1 / \beta} \log \frac{1}{\delta} & \left(\gamma=\frac{1}{\beta}, 0<\delta<\frac{1}{2}\right) . \\ \delta^{1 / \beta} & \left(\gamma>\frac{1}{\beta}\right)\end{cases}
$$

Minor adjustments to the proof of Theorem 5 prove the following result.

THEOREM 8. Let $G$ be a bounded, simply connected open domain. Suppose there exists an $\varepsilon>0$ such that for each $\zeta \in \partial G$ there is a disk, $D_{\zeta}$, of radius $\varepsilon$ with $\zeta \in \partial D_{\zeta}$ and $\bar{D}_{\zeta} \cap G=\phi$. If $u(z)$ is harmonic on $G$, continuous on $\bar{G}$ and has BMOC $\tilde{\omega}(\delta)$, then

$$
\omega(\delta) \leqq \tilde{\omega}(C \delta)+D \delta \int_{\delta}^{E} \frac{\tilde{\omega}(s)}{s^{2}} d s,
$$

where $C, D, E$ are positive constants depending on $G$.

For notational convenience, the "disk condition" described in Theorem 8 will be referred to as a cone condition of order $(1, \varepsilon)$. If $G$ is a bounded, simply connected open domain that does not satisfy a cone condition of any order $(\alpha, \varepsilon)(0<\alpha \leqq 1, \varepsilon>0)$, then we will say $G$ satisfies a cone condition of order $(0,1)$. Thus Theorem 8 shows that if $G$ satisfies a cone condition of order $(1, \varepsilon)$, then our estimates for $\omega(\delta)$ are essentially those given in (2) for the unit disk. This analogy with the disk illustrated further in the following corollaries. 
COROLlary 9. If $G$ satisfies a cone condition of order $(1, \varepsilon)$ $(\varepsilon>0)$, and $\tilde{\omega}(\delta)$ is subadditive, then

$$
\omega(\delta) \leqq C\left(\log \frac{1}{\delta}\right) \tilde{\omega}(\delta) .
$$

CoRollary 10. If $G$ satisfies a cone condition of order $(1, \varepsilon)$ and $\tilde{\omega}(\delta) \leqq \delta^{r}(0<\gamma \leqq 1)$, then

$$
\omega(\delta) \leqq C \begin{cases}\delta^{\gamma} & (0<\delta<1) \\ \delta \log \frac{1}{\delta} & (\gamma=1)\end{cases}
$$

The following corollary improves a result of Dankel [2].

COROLlaRY 11. If $G$ is bounded and convex, and $u(z)$ is harmonic on continuous on $\bar{G}$, then

$$
\omega(\delta) \leqq C \tilde{\omega}(\delta) \log \frac{1}{\delta}
$$

Proof. Since $G$ satisfies a cone condition of order $(1,1)$, it suffices, as in the proof of Corollary 2, to show $\tilde{\omega}(\delta)$ is bounded above and below by multiples of some continuous, nondecreasing concave function $\lambda(\delta)$. The fact that $G$ is a bounded, convex domain implies $\partial G$ is rectifiable, and that $\partial G$ has bounded arc-chord ratio. For $\zeta, \zeta^{\prime} \in \partial G$, let $s\left(\zeta, \zeta^{\prime}\right)$ be the length of the "shorter" arc along $\partial G$ from $\zeta$ to $\zeta$. Then for some constant $A>0$ we have

$$
1 \leqq \frac{s\left(\zeta, \zeta^{\prime}\right)}{\left|\zeta-\zeta^{\prime}\right|} \leqq A
$$

for all $\zeta, \zeta^{\prime} \in \partial G$. Let

$$
\widetilde{\widetilde{\omega}}(\delta)=\sup \left\{\left|u(\zeta)-u\left(\zeta^{\prime}\right)\right|, \zeta, \zeta^{\prime} \in \partial G, s\left(\zeta, \zeta^{\prime}\right) \leqq \delta\right\},
$$

be the BMOC of $u$ with respect to arc length along $\partial G$. Then $\widetilde{\widetilde{\omega}}(\delta)$ is subadditive and for $\delta>0$,

$$
\widetilde{\tilde{\omega}}(\delta) \leqq \tilde{\omega}(\delta) \leqq \widetilde{\tilde{\omega}}(A \delta) \leqq(A+1) \widetilde{\tilde{\omega}}(\delta) .
$$

We now let $\lambda(\delta)$ be a continuous, nondecreasing concave function with $\lambda(\delta) \leqq \widetilde{\widetilde{\omega}}(\delta) \leqq 2 \lambda(\delta)$. This completes the proof.

3. Analytic functions. Let $G$ be a bounded simply connected open domain and suppose $f(z)=u(z)+i v(z)$ is analytic on $G$ and continuous on $\bar{G}$. Using the results from $\S 2$, we obtain results 
relating the MOC of $f$ on $\bar{G}$ to the BMOC of $u$ or $v$.

The following well-known result gives a bound on $\left|f^{\prime}(z)\right|$ in terms of $\omega_{u}(\delta, \bar{G})$.

THEOREM 12. Let $G$ be a bounded region, let $f(z)=u(z)+i v(z)$ be analytic on $G$, and let $u(z)$ be continuous on $\bar{G}$. Then for $z \in G$

$$
\left|f^{\prime}(z)\right| \leqq \frac{2 \omega_{u}\left(d_{z}, \bar{G}\right)}{d_{z}},
$$

where $d_{z}$ denotes the distance from $z$ to $\partial G$.

With proper consideration given to the smoothness of $\partial G$, we can estimate $\omega_{f}(\delta, \bar{G})$ from $(10)$. We first require two definitions.

DEFINITION 13. Let $\lambda(t)(t \geqq 0)$ be a nonnegative, increasing, subadditive function with $\lim _{t \rightarrow 0^{+}} \lambda(t)=0$. A domain $G$ is a $\lambda$-domain if there exists a function $\phi: R \rightarrow R$ and a positive constant $M$ with

$$
G=\{x+i y: y>\phi(x)\},
$$

and

$$
\left|\phi(x)-\phi\left(x^{\prime}\right)\right| \leqq M \lambda\left(\left|x-x^{\prime}\right|\right),
$$

for all $x, x^{\prime} \in R$. The smallest $M$ for which (11) holds is the bound for $G$. Any rotation of $\lambda$-domain is also a $\lambda$-domain.

DeFinition 14. A bounded, simply connected domain $G$ is a local $\lambda$-domain if there exist positive constants $\varepsilon$ and $M$ and a sequence $\left\{U_{i}: i=1,2, \cdots\right\}$ of open sets such that:

(i) For each $\zeta \in \partial G$ there is a $U_{i}$ with $\Delta(\zeta, \varepsilon) \leqq U_{i}$.

(ii) For each $U_{i}$ there is a $\lambda$-domain $G_{i}$ with bound not exceeding $M$ such that:

$$
U_{i} \cap G_{i}=U_{i} \cap G .
$$

$M$ is called a bound for $G$. If $\lambda(x)=C x^{\alpha}$ (some $0<\alpha \leqq 1$ ), then $G$ is a local $\operatorname{Lip}(\alpha)$-domain. Our definition of local Lip(1)-domain coincides with the definition of a domain with minimally smooth boundary [7, p. 189].

The following theorem and its corollary is proved in [4].

THEOREM 15. Let $G$ be a local $\lambda$-domain and let $\mu(t)(t \geqq 0)$ be a nonnegative, increasing, subadditive function with $\lim _{t \rightarrow 0^{+}} \mu(t)=0$. Suppose $f(z)$ is analytic on $G$, continuous on $\bar{G}$ and 


$$
\left|f^{\prime}(z)\right| \leqq \frac{\mu\left(d_{z}\right)}{d_{z}}
$$

for each $z \in G$. Then there is an $\eta>0$ such that

$$
\omega_{f}(\delta, \bar{G}) \leqq A \int_{0}^{\delta} \frac{\mu(t) \lambda^{\prime}(t)}{t} d t,
$$

for all $\delta \leqq \eta$

In (12), we have assumed $\lambda(t)$ is concave and so has a nonnegative derivative at all but at most countably many points. This assumption affects the inequality (12) by at most a constant multiple [5, p. 45].

Corollary 16. Let $G$ be a local Lip $(\alpha)$-domain and let $\beta$ $(0<\beta \leqq 1)$ be given with $\alpha+\beta>1$. If $f(z)$ is continuous on $\bar{G}$, analytic on $G$ and

$$
\left|f^{\prime}(z)\right| \leqq C d_{z}^{\beta-1},
$$

for all $z \in G$, then $f(z)$ satisfies a Lipschitz condition of order $\alpha+$ $\beta-1$ on $\bar{G}$.

Combining Theorems 5, 12 and 15 gives the following result.

THEOREM 17. Suppose $G$ is a local $\lambda$-domain and that $G$ satisfies a cone condition of order $(\alpha, \varepsilon)(0 \leqq \alpha \leqq 1, \varepsilon>0)$. Let $f(z)=u(z)+$ $i v(z)$ be analytic on $G$, continuous on $\bar{G}$ and suppose $u(z)$ has BMOC $\tilde{\omega}_{u}(\delta)$ on $\partial G$. Then there is an $\eta>0$ such that

$$
\omega_{f}(\delta, \bar{G}) \leqq A\left\{\int_{0}^{\delta} \frac{\lambda^{\prime}(t)}{t}\left[\tilde{\omega}(C t)+D t^{1 / \beta} \int_{t}^{E} \frac{\tilde{\omega}(s)}{s^{(1+\beta) / \beta}} d s\right] d t\right\},
$$

where $\beta=z-\alpha$ and $A, C, D, E$ are constants depending on $G$.

In Theorem 17, we have again assumed $\lambda(t)$ is concave. The proof is immediate since the representation (9) for our estimate of $\omega_{u}(\delta, \bar{G})$ is clearly nonnegative, increasing, subadditive and tends to 0 with $\delta$. Corollary 16 can be used to draw analogous conclusions concerning Lipschitz conditions.

4. Examples and remarks. In this section we first present a class of examples that shows Corollaries 2 and 6 are best possible in the sense that the exponents on the $\delta$ 's cannot be improved. Let $1<\beta \leqq 2$ and let $\phi_{\beta}(z)=(1-z)^{\beta}$ where, for $1<\beta<2$, we take a 
branch cut along $z>1$. Then $\phi_{\beta}$ is $1-1$ and continuous on $\bar{D}$, analytic on $D$ onto a domain $G_{\beta}$ that satisfies a cone condition of order $(2-\beta, 1)$. Consider the function $u_{\beta}(\zeta)$, harmonic on $G_{\beta}$, continuous on $\bar{G}_{\beta}$ with $u_{\beta}(\zeta)=|\zeta|$ for $\zeta \in \partial G_{\beta}$. Define $v_{\beta}(z)$ on $\bar{D}$ by $v_{\beta}(z)=$ $u_{\beta}\left((1-z)^{\beta}\right)$. Then $v_{\beta}$ is continuous on $\bar{D}$, harmonic on $D$ and $u_{\beta}(\zeta)=$ $v_{\beta}\left(\phi_{\beta}^{-1}(\zeta)\right)\left(\zeta \in G_{\beta}\right)$, where $\phi_{\beta}^{-1}(\zeta)=1-\zeta^{1 / \beta}$ is defined with branch cut along $\zeta<0$.

Now $\tilde{\omega}_{u_{\beta}}\left(\delta, \partial G_{\beta}\right)=\delta$. If $\delta$ is given with $0<\delta<2^{\beta}$, then $\delta \in G_{\beta}$ and

$$
\begin{aligned}
\omega_{u_{\beta}}\left(\delta, \bar{G}_{\beta}\right) \geqq u_{\beta}(\delta) & =v_{\beta}\left(1-\delta^{1 / \beta}\right) \\
& =\frac{1}{2 \pi} \int_{-\pi^{\beta}}^{\pi_{v}}\left(e^{i \theta}\right) P\left(1-\delta^{1 / \beta}, \theta\right) d \theta \\
& \geqq \frac{1}{\pi} \int_{\delta^{1 / \beta}}^{\pi}\left|1-e^{i \theta}\right|^{\beta} P\left(1-\delta^{1 / \beta}, \theta\right) d \theta
\end{aligned}
$$

For $\delta^{1 / \beta} \leqq \theta \leqq \pi$ we have $\left|1-e^{i \theta}\right|^{\beta} \geqq(2 \theta / \pi)^{\beta}$ and $P\left(1-\delta^{1 / \beta}, \theta\right) \geqq\left(\delta^{1 / \beta} \mid \theta^{2}\right)$. Thus

$$
\begin{aligned}
\omega_{u_{\beta}}\left(\delta, \bar{G}_{\beta}\right) & \geqq \frac{2^{\beta} \delta^{1 / \beta}}{\pi^{1+\beta}} \int_{\tilde{\delta}^{1 / \beta}}^{\pi} \theta^{\beta-2} d \theta \\
& \geqq\left(\frac{2^{\beta-1}}{\pi^{2}(\beta-1)}\right) \delta^{1 / \beta} \\
& =C \delta^{(1-\beta) / \beta} \tilde{\omega}_{u_{\beta}}(\delta, \partial G) .
\end{aligned}
$$

The example further shows that the constant in Corollary 6 cannot be taken independent of $\alpha=2-\beta$. A similar argument, using $\phi_{\beta}(z)=A(1-z)^{\beta}(A>0)$ shows the constants in Corollaries 2 and 6 cannot be taken independent of $|G|$. If we take $0<\gamma \leqq 1$ and repeat the above argument with $u_{\beta}(\zeta)=|\zeta|^{r}\left(\zeta \in \partial G_{\beta}\right)$, we obtain examples that show Corollaries 3 and 7 are best possible.

As a final remark, we note that Theorem 5 actually says something about were in $\bar{G}|u(\zeta)-u(z)|$ can achieve the bound given in (5).

THEOREM 18. Let $0 \leqq \alpha_{1}<\alpha_{2} \leqq 1$ and $\varepsilon_{1}, \varepsilon_{2}, \delta_{0}>0$ be given (if $\alpha_{1}=0$, take $\left.\varepsilon_{1}=1\right)$. Let $G$ satisfy a cone condition of order $\left(\alpha_{1}, \varepsilon_{1}\right)$ and suppose there is $a \gamma \subseteq \partial G$ such that $G$ satisfies a cone condition of order $\left(\alpha_{2}, \varepsilon_{2}\right)$ at each $\zeta \in \gamma$. Suppose $u(z)$ is harmonic on $G$, continuous on $\bar{G}$ and has BMOC $\tilde{\omega}(\delta)$. If, for each $0<\delta \leqq \delta_{0}$ we have $\omega(\delta)=\mid u(\zeta)-u(z)$ for some $\zeta \in \gamma, z \in \bar{G},|\zeta-z| \leqq \delta$, then

$$
\omega(\delta) \leqq \tilde{\omega}(C \delta)+D \delta^{1 / \beta_{2}} \int_{\delta}^{E} \frac{\tilde{\omega}(s)}{s^{\left(1+\beta_{2}\right) / \beta_{2}}} d s \quad\left(\delta \leqq \delta^{0}\right)
$$


where $\beta_{2}=2-\alpha_{2}$ and $C, D, E$ are positive constants depending on $\alpha_{2}, \varepsilon_{2}$ and $|G|$.

Referring back to the example presented at the beginning of this section, Theorem 17 gives the following fact. Let $\left\{\delta_{n}\right\}_{n=1}^{\infty}$ be a sequence of positive numbers with $\delta_{n} \rightarrow 0$. Suppose $\zeta_{n}, z_{n}(n=1,2, \cdots)$ are given with $\zeta_{n} \in \partial G_{\beta}, \quad z_{n} \in \bar{G}_{\beta},\left|\zeta_{n}-z_{n}\right| \leqq \delta_{n}$ and $\omega_{u_{\beta}}\left(\delta_{n}, \bar{G}_{\beta}\right)=$ $\left|u_{\beta}\left(\zeta_{n}\right)-u_{\beta}\left(z_{n}\right)\right|$. Then $\lim \zeta_{n}=\lim z_{n}=0$.

\section{REFERENCES}

1. A. Beurling, Études sur un Problème de Majoration, Almquist and Wiksell, Upsala, 1933.

2. T. Dankel, On moduli of continuity of analytic and harmonic functions, Rocky Mountain J. Math., 9 (1979), 519-526.

3. G. H. Hardy and J. E. Littlewood, Some properties of fractional integrals, II, Math. Zeit., 34 (1931), 403-439.

4. E. Johnston, Growth of derivatives and the modulus of continuity of analytic functions, Rocky Mountain J. Math., 9 (1979), 671-681.

5. G. G. Lorentz, Approximation of Functions, New York, Holt, Rinehart and Winston, 1966.

6. L. A. Rubel, B. A. Taylor, A. L. Shields, Mergelyan sets and the modulus of continu. ity of analytic functions, J. Approximation Theory, 15 (1975), 23-39.

7. E. Stein, Singular Integrals and Differentiability Properties of Functions, Princeton, Princeton University Press, 1970.

Received June, 21978.

Iowa State University

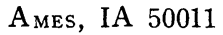




\section{PACIFIC JOURNAL OF MATHEMATICS}

\section{EDITORS}

DONALD BABBITT (Managing Editor)

University of Galifornia

Los Angeles, California 90024

HUGO RossI

University of Utah

Salt Lake City, UT 84112

C. C. MOORE AND ANDREW OGG

University of California

Berkeley, CA 94720
J. DUGUNDJI

Department of Mathematics University of Southern California Los Angeles, California 90007

R. FINN AND J. MILGRAM Stanford University

Stanford, California 94305

ASSOCIATE EDITORS

E. F. BECKENBACH

B. H. NeumanN

F. WOLF

K. YosHidA

\section{SUPPORTING INSTITUTIONS}

UNIVERSITY OF BRITISH COLUMBIA UNIVERSITY OF SOUTHERN CALIFONIA CALIFORNIA INSTITUTE OF TECHNOLOGY UNIVERSITY OF CALIFORNIA MONTANA STATE UNIVERSITY STANFORD UNIVERSITY UNIVERSITY OF HAWAII UNIVERSITY OF NEVADA, RENO UNIVERSITY OF TOKYO U'NIVERSITY OF UTAH NEW MEXICO STATE UNIVERSITY WASHINGTON STATE UNIVERSITY OREGON STATE UNIVERSITY UNIVERSITY OF OREGON UNIVERSITY OF WASHINGTON 


\section{Pacific Journal of Mathematics}

\section{Vol. 90, No. $1 \quad$ September, 1980}

Shashi Prabha Arya and M. K. Singal, On the locally countable sum

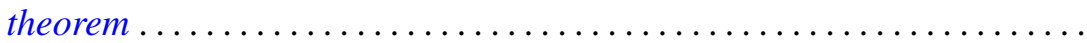

John Theodore Baldwin and David William Kueker, Ramsey quantifiers and

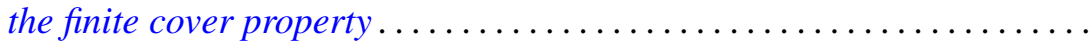

Richard Body and Roy Rene Douglas, Unique factorization of rational

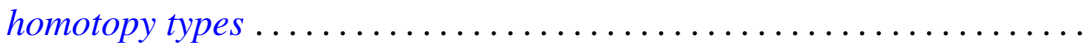

Ethan Bolker and Ben G. Roth, When is a bipartite graph a rigid

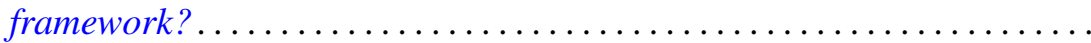

Alicia B. Winslow, Continua in the Stone-Čech remainder of $R^{2} \ldots \ldots \ldots$

Richard D. Carmichael and Elmer Kinji Hayashi, Analytic functions in tubes which are representable by Fourier-Laplace integrals ..............

Stephen D. Cohen, The Galois group of a polynomial with two indeterminate coefficients ..............................

Russell Allan Johnson, Strong liftings commuting with minimal distal

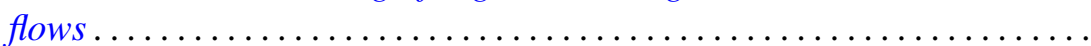

Elgin Harold Johnston, The boundary modulus of continuity of harmonic

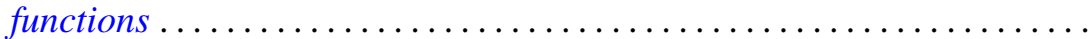

Akio Kawauchi and Takao Matumoto, An estimate of infinite cyclic

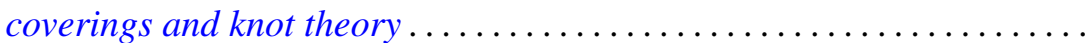

Keith Milo Kendig, Moiré phenomena in algebraic geometry: rational alternations in $\mathbf{R}^{2}$...

Roger T. Lewis and Lynne C. Wright, Comparison and oscillation criteria for selfadjoint vector-matrix differential equations .

Teck Cheong Lim, Asymptotic centers and nonexpansive mappings in conjugate Banach spaces .......................

David John Lutzer and Robert Allen McCoy, Category in function spaces.

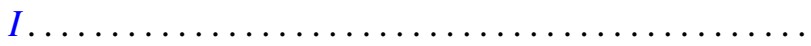

Richard A. Mollin, Induced p-elements in the Schur group ...

Jonathan Simon, Wirtinger approximations and the knot groups of $F^{n}$ in $S^{n+2}$

Robert L. Snider, The zero divisor conjecture for some solvable groups...

H. M. (Hari Mohan) Srivastava, A note on the Konhauser sets of biorthogonal polynomials suggested by the Laguerre polynomials...

Nicholas Th. Varopoulos, A probabilistic proof of the Garnett-Jones theorem on BMO.

Frank Arvey Wattenberg, $[0, \infty]$-valued, translation invariant measures on $N$ and the Dedekind completion of ${ }^{*} R \ldots \ldots \ldots \ldots . .$. 